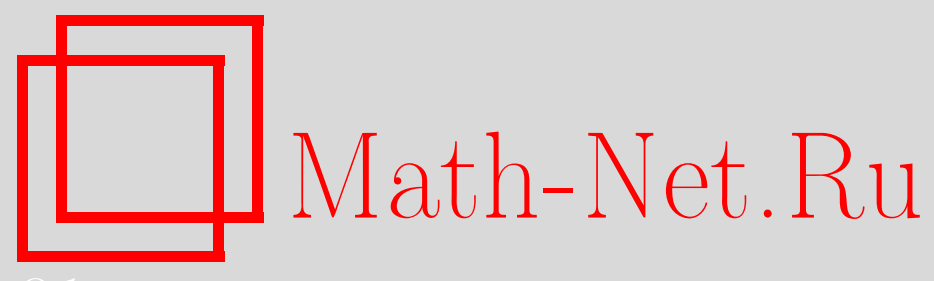

M. В. Заец, Построение подстановок с использованием вариационно-координатно полиномиальных функций над примарным кольцом вычетов, Матем. вопр. криптогр., 2015, том 6, выпуск 1, 5-32

DOI: https://doi.org/10.4213/mvk149

Использование Общероссийского математического портала Math-Net.Ru подразумевает, что вы прочитали и согласны с пользовательским соглашением http://www.mathnet.ru/rus/agreement

Параметры загрузки:

IP : 18.208 .226 .222

26 апреля 2023 г., 11:26:14

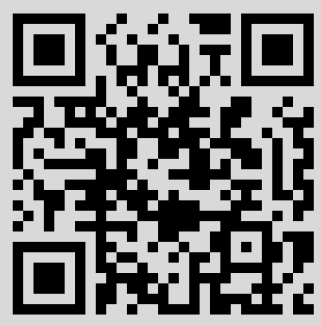




\title{
Построение подстановок с использованием вариационно-координатно полиномиальных функций над примарным кольцом вычетов
}

\author{
М. В. Заец \\ ФГУП Научно-исследовательский институт «Квант», Москва
}

Получено 19.ХІ.2014

В статье рассматриваются способы построения подстановок над примарным кольцом вычетов, использующие вариационно-координатно полиномиальные функции. Данная работа обобщает известные результаты о подстановочных полиномиальных функциях, полиномиальных $n$-квазигруппах и биективных полиномиальных вектор-функциях.

Ключевые слова: n-квазигруппы, подстановочные полиномиальные функции, биективные полиномиальные вектор-функции, вариационно-координатно полиномиальные функции.

Construction of substitutions by means of variationally-coordinate polynomial functions over the primary residue ring

M. V. Zaets

FSUE Scientific Research Institute «Kvant», Moscow

Abstract. We consider methods permitting to construct substitutions over the primary residue ring by means of variationally-coordinate polynomial functions. Our paper generalizes known results on invertible polynomial functions, polynomial $n$-quasigroups and bijective polynomial vectorial functions.

Key words: $n$-quasigroups, invertible polynomial functions, bijective polynomial functions, variationally-coordinate polynomial functions.

Citation: Mathematical Aspects of Cryptography, 2015, vol. 6, no. 1, pp. 5-32 (Russian) 


\section{§ 1. Биективные полиномиальные функции над примарным кольцом вычетов}

В этом параграфе приводятся известные результаты о биективных полиномиальных функциях над примарным кольцом вычетов, которые будут далее обобщены на более широкий класс функций. Класс всех полиномиальных функций от $n \in \mathbb{N}$ переменных над кольцом вычетов $\mathbb{Z}_{p^{m}}$ будем обозначать через $\mathcal{P}_{p^{m}}(n)$, а функции от переменных $x_{1}, \ldots, x_{n}$ записывать кратко $f(\mathbf{x})=f\left(x_{1}, \ldots, x_{n}\right)$.

Для многочлена $f(\mathbf{x}) \in \mathbb{Z}_{p^{m}}\left[x_{1}, \ldots, x_{n}\right]$ определена формальная частная производная по переменной $x_{i}, i \in \overline{1, n}$ (см., например, [3]).

Определение 1. Пусть задан многочлен

$$
f(\boldsymbol{x})=\sum_{\left(l_{1}, \ldots, l_{n}\right) \in \mathbb{N}_{0}^{n}} a_{l_{1}, \ldots, l_{n}} x_{1}^{l_{1}} \ldots x_{n}^{l_{n}}, a_{l_{1}, \ldots, l_{n}} \in \mathbb{Z}_{p^{m}},
$$

и $i \in \overline{1, n}$. Тогда многочлен $\frac{\partial f}{\partial x_{i}} \in \mathbb{Z}_{p^{m}}\left[x_{1}, \ldots, x_{n}\right]$, определяемый по правилу

$$
\frac{\partial f}{\partial x_{i}}(\boldsymbol{x})=\sum_{\left(l_{1}, \ldots, l_{n}\right) \in \mathbb{N}_{0}^{n}, l_{i} \geq 1} l_{i} a_{l_{1}, \ldots, l_{n}} x_{1}^{l_{1}} \ldots x_{i}^{l_{i}-1} \ldots x_{n}^{l_{n}}
$$

называется его формальной частной производной по переменной $x_{i}$.

Формальная частная производная по переменной также является многочленом, и поэтому ее можно рассматривать как полиномиальную функцию.

Напомним, что полиномиальной вектор-функцией называется отображение $F=\left(f_{1}, \ldots, f_{l}\right): \mathbb{Z}_{p^{m}}^{n} \rightarrow \mathbb{Z}_{p^{m}}^{l}, l \in \mathbb{N}$, где $f_{i} \in \mathcal{P}_{p^{m}}(n), i=\overline{1, l}$.

Определение 2. Для многочлена $f(\mathbf{x}) \in \mathbb{Z}_{p^{m}}\left[x_{1}, \ldots, x_{n}\right]$ полиномиальную вектор-функцию $\operatorname{grad} f(\mathbf{x})=\left(\frac{\partial f}{\partial x_{1}}(\mathbf{x}), \ldots, \frac{\partial f}{\partial x_{n}}(\mathbf{x})\right)$, составленную из его формальных частных производных по всем переменным, будем называть градиентом многочлена $f(\mathbf{x})$.

Напомним ([8,9]), что пара $(Q, f)$, где $f: Q^{n} \rightarrow Q$ и $Q-$ конечное множество, называется $n$-квазигруппой (или $n$-арной квазигруппой), если 
любая унарная операция, получаемая фиксацией всех аргументов операции $f$, кроме одного, любыми значениями из $Q$, является биекцией. Также иногда $n$-квазигруппой называют саму функцию $f$. Если $n=1$, то $n$-квазигруппа - это подстановка элементов множества $Q$. Если $Q$ - кольцо, и функция $f$ является полиномиальной над $Q$, то такая $n$-квазигруппа также называется полиномиальной. Опишем полиномиальные функции, задающие $n$-квазигруппу на кольце $\mathbb{Z}_{p^{m}}$. Для этого приведем критерий биективности полиномиального преобразования кольца вычетов $\mathbb{Z}_{p^{m}}$, который справедлив и для более широкого класса колец (см., например, $[2,4,5,6$, $13]$ ); такие функции задают подстановку на кольце $\mathbb{Z}_{p^{m}}$, и их называют подстановочными многочленами.

Для любой полиномиальной функции $f(\mathbf{x})$ над кольцом $\mathbb{Z}_{p^{m}}$ очевидным образом определяется функция $f(\mathbf{x})\left(\bmod p^{k}\right), k \in \overline{1, m}$, получаемая путем приведения коэффициентов некоторого полиномиального представления $f(\mathbf{x})$ по модулю $p^{k}$ и рассматриваемая над кольцом вычетов $\mathbb{Z}_{p^{k}}$. Ясно, что такое определение корректно и не зависит от представления функции $f(\mathbf{x})$, так как $f(\mathbf{x})$ сохраняет отношение сравнимости по модулю $p^{k}$. В частности, $f(\mathbf{x})(\bmod p)$ является функцией над полем $\mathbb{Z}_{p}$.

Теорема 1 ([13]). Функиия $f(x) \in \mathcal{P}_{p^{m}}(1), m>1$, задает биективное преобразование (подстановку) кольца $\mathbb{Z}_{p^{m}}$ тогда и только тогда, когда одновременно выполняются следующие условия:

1) $f(x)(\bmod p)$ задает биективное преобразование поля $\mathbb{Z}_{p}$;

2) $f^{\prime}(x)(\bmod p)$ не имеет корней в поле $\mathbb{Z}_{p}$.

Данный критерий справедлив в общем случае и для конечных коммутативных локальных колец $R$, где в качестве $\mathbb{Z}_{p}$ берется поле вычетов кольца $\bar{R}=R / J(R)$, где $J(R)$ - его радикал Джекобсона.

Сформулируем приведенный в [6] критерий того, что полиномиальная функция $f(\mathbf{x}) \in \mathcal{P}_{p^{m}}(n)$ задает $n$-квазигруппу.

Tеорема 2 ([6]). Функциия $f(\mathbf{x}) \in \mathcal{P}_{p^{m}}(n), m>1$, задает n-квазигруппу $(n>1)$ на кольие $\mathbb{Z}_{p^{m}}$ тогда и только тогда, когда для любых 
$\left(a_{1}, \ldots, a_{n-1}\right) \in \mathbb{Z}_{p}^{n-1} \kappa$ каждая из полиномиальных функций:

$$
\begin{gathered}
f_{1}\left(x_{1}\right)=f\left(x_{1}, a_{1}, \ldots, a_{n-1}\right), \\
f_{2}\left(x_{2}\right)=f\left(a_{1}, x_{2}, \ldots, a_{n-1}\right), \\
\vdots \\
f_{n}\left(x_{n}\right)=f\left(a_{1}, \ldots, a_{n-1}, x_{n}\right)
\end{gathered}
$$

задает биекцию кольцуа $\mathbb{Z}_{p^{m}}$.

Используя данную теорему и теорему 1 , очевидным образом получим критерий того, что функция $f(\mathbf{x}) \in \mathcal{P}_{p^{m}}(n)$ задает $n$-квазигруппу на $\mathbb{Z}_{p^{m}}$. Доказательство данного критерия опустим, поскольку позднее докажем его для более широкого класса функций.

Теорема 3. Функиия $f(\mathbf{x}) \in \mathcal{P}_{p^{m}}(n), m>1$, задает $n$-квазигруппу на кольце $\mathbb{Z}_{p^{m}}$ тогда и только тогда, когда выполняются следующие условия:

1) $f(\mathbf{x})(\bmod p)$ задает п-квазигруппу на $\mathbb{Z}_{p}$;

2) $\operatorname{grad} f(\alpha)(\bmod p)$ не содержит нулевых координат при любом $\alpha \in \mathbb{Z}_{p}^{n}$.

Условие 2) приведенной теоремы равносильно тому, что для любого $i \in \overline{1, n}$ формальная частная производная $\frac{\partial f}{\partial x_{i}}(\mathbf{x})(\bmod p)$ не обращается в нуль в $\mathbb{Z}_{p}^{n}$.

Пример 1. Рассмотрим многочлен от двух переменных над кольцом $\mathbb{Z}_{p^{m}}$ следующего вида:

$$
f(x, y)=a_{1} x+a_{2} x^{k} y^{l}+a_{3} y, k, l \in \mathbb{N}, a_{1}, a_{3} \in \mathbb{Z}_{p^{m}}^{*}, a_{2} \in D\left(\mathbb{Z}_{p^{m}}\right),
$$

где $\mathbb{Z}_{p^{m}}^{*}$ - мультипликативная группа кольца вычетов $\mathbb{Z}_{p^{m}}$, а $D\left(\mathbb{Z}_{p^{m}}\right)$ - множество делителей нуля в $\mathbb{Z}_{p^{m}}$, или, что то же самое, множество необратимых элементов в $\mathbb{Z}_{p^{m}}$. Тогда его формальные частные производные по переменным $x, y$ равны соответственно:

$$
\frac{\partial f}{\partial x}(x, y)=a_{1}+k a_{2} x^{k-1} y^{l}, \frac{\partial f}{\partial y}(x, y)=a_{3}+l a_{2} x^{k} y^{l-1} \text {. }
$$

Так как $a_{1}, a_{3} \in \mathbb{Z}_{p^{m}}^{*}, a_{2} \in D\left(\mathbb{Z}_{p^{m}}\right)$, то $\left(a_{1}, p\right)=\left(a_{3}, p\right)=1$ и $p \mid a_{2}$. Градиент многочлена $f(x, y)$ после приведения по модулю $p$ примет вид (в силу 
делимости $\left.p \mid a_{2}\right)$ :

$$
\operatorname{grad} f(x, y) \equiv\left(a_{1}, a_{3}\right)(\bmod p) .
$$

Вектор $\left(a_{1}, a_{3}\right)$ не содержит нулевых компонент. Приведя $f(x, y)$ по модулю $p$, получим:

$$
f(x, y) \equiv a_{1} x+a_{3} y(\bmod p), a_{i} \not \equiv 0(\bmod p), i \in\{1,3\} .
$$

Таким образом, $f(x, y)(\bmod p)$ - линейная функция над полем $\mathbb{Z}_{p}$. Очевидно, что она задает 2-квазигруппу на $\mathbb{Z}_{p}$ и что для многочлена $f(x, y)$ выполнены все условия теоремы 3 , а значит, $f(x, y)$ задает 2-квазигруппу на кольце $\mathbb{Z}_{p^{m}}$ при любых указанных выше параметрах.

Напомним, что 2-квазигруппы называют просто квазигруппами, и их таблицы Кэли являются латинскими квадратами. В частности, данный пример показывает способ построения целого класса латинских квадратов.

Пусть $\left(f_{1}(\mathbf{x}), \ldots, f_{t}(\mathbf{x})\right), t \in \mathbb{N}$, - система многочленов над кольцом $\mathbb{Z}_{p^{m}}$ от $n$ переменных. Она задает полиномиальную вектор-функцию $F: \mathbb{Z}_{p^{m}}^{n} \rightarrow \mathbb{Z}_{p^{m}}^{t}$, которую будем обозначать $F=\left(f_{1}, \ldots, f_{t}\right)$. Напомним, что матрица, составленная из формальных частных производных многочленов $f_{1}, \ldots, f_{t}$ по всем переменным, имеет вид $\left(\begin{array}{c}\operatorname{grad} f_{1}(\mathbf{x}) \\ \vdots \\ \operatorname{grad} f_{t}(\mathbf{x})\end{array}\right)$ и называется матрицей Якоби системы многочленов $F=\left(f_{1}, \ldots, f_{t}\right)$ в точке $\mathbf{x} \in \mathbb{Z}_{p^{m}}^{n}$. Будем обозначать ее всюду далее через $\mathcal{J}_{F}(\mathbf{x})$. Определитель матрицы Якоби $\operatorname{det} \mathcal{J}_{F}(\mathbf{x})=\left|\mathcal{J}_{F}(\mathbf{x})\right|$ является полиномиальной функцией и называется якобианом системы многочленов $F=\left(f_{1}, \ldots, f_{t}\right)$.

Результаты о биективности полиномиальной функции от одной переменной обобщаются на случай полиномиальной вектор-функции $F(\mathbf{x})=\left(f_{1}(\mathbf{x}), \ldots, f_{n}(\mathbf{x})\right)$ от $n$ переменных теоремой 4 (см. [2, 3, 7]), которую также можно сформулировать для конечных коммутативных локальных колец. Далее для полиномиальной вектор-функции $F(\mathbf{x})=\left(f_{1}(\mathbf{x}), \ldots, f_{n}(\mathbf{x})\right)$ через $F(\mathbf{x})(\bmod p)$ будем обозначать вектор-функцию над полем $\mathbb{Z}_{p}$, определяемую путем приведения каждой компоненты $f_{i}(\mathbf{x}), i=\overline{1, n}$, по модулю $p$. 
Теорема 4 ([3]). Полиномиальная вектор-функция $F=\left(f_{1}, \ldots, f_{n}\right)$ : $\mathbb{Z}_{p^{m}}^{n} \rightarrow \mathbb{Z}_{p^{m}}^{n}, m>1$, задает биекцию тогда и только тогда, когда выполняются следуюшие условия:

1) $F(\mathbf{x})(\bmod p)$ задает биекциию на $\mathbb{Z}_{p}^{n}$;

2) якобиан $\left|\mathcal{J}_{F}(\alpha)\right|(\bmod p)$ отличен от нуля при всех $\alpha \in \mathbb{Z}_{p}^{n}$.

Далее будем говорить, что наборы целых чисел $\alpha=\left(a_{1}, \ldots, a_{n}\right)$ и $\beta=\left(b_{1}, \ldots, b_{n}\right)$ сравнимы по модулю $d$ (или $\left.\alpha \equiv \beta(\bmod d)\right)$, если $a_{i} \equiv b_{i}(\bmod d)$ для всех $i \in \overline{1, n}$.

Сформулируем утверждение о равновероятности полиномиальной функции (в литературе наряду с этим используют термины сбалансированность, равномерность).

Определение 3. Функция $f: \mathbb{Z}_{p^{m}}^{n} \rightarrow \mathbb{Z}_{p^{m}}$ называется равновероятной, если для любых $a, b \in \mathbb{Z}_{p^{m}}$ верно равенство $\left|f^{-1}(a)\right|=\left|f^{-1}(b)\right|$.

Это равносильно тому, что для любого $a \in \mathbb{Z}_{p^{m}}$ :

$$
\left|f^{-1}(a)\right|=p^{m(n-1)} .
$$

Для полиномиальных функций справедливо следующее достаточное условие равноверояности (см. $[1,2])$, которое в общем случае можно сформулировать и для конечных коммутативных локальных колец.

Утверждение 5 ([2]). Пусть $f(\mathbf{x}) \in \mathcal{P}_{p^{m}}(n), m>1$, и выполнены следующие условия:

1) $f(\mathbf{x})(\bmod p)-$ равновероятная функция над полем $\mathbb{Z}_{p}$;

2) $\operatorname{grad} f(\alpha) \not \boldsymbol{\theta}(\bmod p)$ при любом $\alpha \in \mathbb{Z}_{p}^{n}$, где $\boldsymbol{\theta}$ - нулевой вектор.

Тогда функиия $f(\mathbf{x})$ равновероятна.

\section{§ 2. Биективные ВКП-функции над примарным кольцом вычетов}

Любой элемент $a$ примарного кольца вычетов $\mathbb{Z}_{p^{m}}$, где $m \in \mathbb{N}, p-$ простое, можно однозначно представить в виде

$$
a=a^{(0)}+p \cdot a^{(1)}+\ldots+p^{m-1} \cdot a^{(m-1)}, j=\overline{0, m-1},
$$


где $a^{(j)} \in \mathcal{B}=\{0, \ldots, p-1\} \subset \mathbb{Z}_{p^{m}}$, называемом разложением элемента $a$ в $p$-ичном координатном множестве $\mathcal{B}$. Отображения

$$
\gamma_{j}: \mathbb{Z}_{p^{m}} \rightarrow \mathcal{B}, \quad \gamma_{j}(a)=a^{(j)}, j=\overline{0, m-1},
$$

называются координатными функциями в координатном множестве $\mathcal{B}$, а элементы $a^{(j)} \in \mathcal{B}$ - координатами $j$-го порядка элемента $a$ в координатном множестве $\mathcal{B}$. В частности, любой вектор $\mathbf{x}=\left(x_{1}, \ldots, x_{n}\right) \in \mathbb{Z}_{p^{m}}^{n}$ однозначно представляется в виде конечной суммы:

$$
\mathbf{x}=\mathbf{x}^{(0)}+p \cdot \mathbf{x}^{(1)}+\ldots+p^{m-1} \cdot \mathbf{x}^{(m-1)},
$$

где $\mathbf{x}^{(j)}=\left(x_{1}^{(j)}, \ldots, x_{n}^{(j)}\right) \in \mathcal{B}^{n}, j=\overline{0, m-1}$.

Если ввести на $\mathcal{B}$ операции сложения « $\oplus »$ и умножения «®» по правилу

$$
a \oplus b=\gamma_{0}(a+b), a \otimes b=\gamma_{0}(a \cdot b), \quad a, b \in \mathcal{B},
$$

то алгебра $(\mathcal{B}, \oplus, \otimes) \cong \mathbb{Z}_{p^{m}} / p \mathbb{Z}_{p^{m}} \cong \mathbb{Z}_{p}$ будет являться полем из $p$ - элементов.

Определение 4. Для функции $f(\mathbf{x}): \mathbb{Z}_{p^{m}}^{n} \rightarrow \mathbb{Z}_{p^{m}}$ и $j \in \overline{0, m-1}$ отображение $\gamma_{j} f: \mathbb{Z}_{p^{m}}^{n} \rightarrow \mathcal{B}$, определяемое по правилу

$$
\left(\gamma_{j} f\right)(\alpha)=\gamma_{j}(f(\alpha))
$$

для всех $\alpha \in \mathbb{Z}_{p^{m}}^{n}$, будем называть ее $j$-й координатной функиией, или $j-м$ координатным отображением.

Другими словами, любая функция $f(\mathbf{x}): \mathbb{Z}_{p^{m}}^{n} \rightarrow \mathbb{Z}_{p^{m}}$ представима следующим образом через свои координатные функции:

$$
f(\mathbf{x})=\sum_{j=0}^{m-1} p^{j} \cdot \gamma_{j} f(\mathbf{x})
$$

При этом любую координатную функцию $\gamma_{j} f, j=\overline{0, m-1}$, можно рассматривать как функцию $\gamma_{j} f: \mathcal{B}^{n m} \rightarrow \mathcal{B}$ от $n m$ переменных над полем $\mathcal{B}$, в роли которых выступают координаты переменных $\mathbf{x}^{(0)}, \ldots, \mathbf{x}^{(m-1)}$. В таком случае будем предполагать, что координаты переменных расположены в указанном 
порядке, т. е. $\gamma_{j} f=\gamma_{j} f\left(\mathbf{x}^{(0)}, \ldots, \mathbf{x}^{(m-1)}\right)$. Следовательно, любая такая координатная функция может быть представлена многочленом над полем $\mathcal{B}$ от указанных переменных.

Определение 5. Функцию $f(\mathbf{x}): \mathbb{Z}_{p^{m}}^{n} \rightarrow \mathbb{Z}_{p^{m}}, m \in \mathbb{N}$ назовем вариационно-координатно полиномиальной (или ВКП-функиией), если для любого $j \in \overline{0, m-1}$ существует полиномиальная функция $p_{j}(\mathbf{x}) \in \mathcal{P}_{p^{m}}(n), j$-я координатная функция которой совпадает с $j$-й координатной функцией функции $f(\mathbf{x})$, т. е. выполняется равенство

$$
\gamma_{j} f(\mathbf{x})=\gamma_{j} p_{j}(\mathbf{x}), j=\overline{0, m-1} .
$$

Класс всех ВКП-функций от $n$ переменных над $\mathbb{Z}_{p^{m}}$ обозначим через $\mathcal{C P}_{p^{m}}(n)$. Класс ВКП-функций был введен автором и описывался подробно в работах $[11,12,14]$.

Поясним введенное определение. Произвольная функция $f(\mathbf{x}) \in$ $\in \mathcal{F}_{p^{m}}(n)$ является вариационно-координатно полиномиальной, если существуют такие многочлены (полиномиальные функции) $p_{0}(\mathbf{x}), p_{1}(\mathbf{x}), \ldots$, $p_{m-1}(\mathbf{x})$ над кольцом $\mathbb{Z}_{p^{m}}$, что равенство

$$
f(\alpha)=\sum_{j=0}^{m-1} p^{j} \cdot \gamma_{j} p_{j}(\alpha)
$$

выполнено для всех $\alpha \in \mathbb{Z}_{p^{m}}^{n}$. Будем говорить, что $p_{j}(\mathbf{x})$ является многочленом $j$-й координаты функции $f(\mathbf{x})$ или ее $j$-м координатным многочленом (отметим, что данный многочлен для функции $f(\mathbf{x})$ определяется неоднозначно). Использование в этом термине слова «вариационно» подчеркивает тот факт, что данные координатные многочлены могут быть разными для различных координат.

Из определения очевидным образом следует, что при $m=1$ классы ВКП и полиномиальных функций совпадают. А при $m>1$ справедливо включение

$$
\mathcal{P}_{p^{m}}(n) \subseteq \mathcal{C} \mathcal{P}_{p^{m}}(n) .
$$

В дальнейшем, если не оговорено иное, считаем, что $m>1$. 
Пример 2. Зададим ВКП-функцию $f(x)$ от одной переменной над $\mathbb{Z}_{8}$ координатными многочленами

$$
p_{0}(x)=p_{1}(x)=x, p_{2}(x)=7 x .
$$

Таблица значений $f(x)$ представлена ниже.

Таблица 1

\begin{tabular}{|l|l|l|l|l|l|l|l|l|}
\hline$x$ & 0 & 1 & 2 & 3 & 4 & 5 & 6 & 7 \\
\hline$f(x)$ & 0 & 5 & 6 & 7 & 4 & 1 & 2 & 3 \\
\hline
\end{tabular}

Данная ВКП-функция является полиномиальной, однако любое ее полиномиальное представление имеет степень не меньше 3 (доказывается непосредственной проверкой). В частности, одно из таких представлений имеет вид $2 x^{3}+3 x$.

Сформулируем без доказательства основные свойства ВКП-функций, которые далее будем использовать [14].

Теорема 6. Справедливы утверждения:

1) для любого $n \in \mathbb{N}$ классы полиномиальных и ВКП-функиий над $\mathbb{Z}_{p^{2}}$ от $n$ переменных совпадают, m. е. $\mathcal{P}_{p^{2}}(n)=\mathcal{C P}_{p^{2}}(n)$;

2) для любых $n \in \mathbb{N}$ и $m \geq 3$ класс ВКП-функиий $\mathcal{C P}_{p^{m}}(n)$ не совпадает с классом полиномиальных $\mathcal{P}_{p^{m}}(n)$.

Теорема 7. Пусть функция $f(\mathbf{x}) \in \mathcal{C} \mathcal{P}_{p^{m}}(n), m \geq 3, u p_{0}(\mathbf{x}), \ldots, p_{m-1}(\mathbf{x})$ ее координатные многочлены. Если существуют такие $i, j \in \overline{0, m-1}(i \neq j)$ u $\alpha \in \mathcal{B}^{n}$, что

$$
\operatorname{grad} p_{i}(\alpha) \not \operatorname{grad} p_{j}(\alpha)(\bmod p),
$$

mo $f(\mathbf{x}) \notin \mathcal{P}_{p^{m}}(n)$.

Теорема 8 (формула Тейлора). Если функиия $f(\mathbf{x}) \in \mathcal{C P}_{p^{m}}(n)$ и $p_{0}(\mathbf{x}), \ldots, p_{m-1}(\mathbf{x})$ - ее координатныле многочлены, то для любых $j \in \overline{0, m-1} u \mathbf{h}=\left(h_{1}, \ldots, h_{n}\right) \in \mathbb{Z}_{p^{m}}^{n}$ справедливо сравнение:

$$
f\left(\mathbf{x}+p^{j} \cdot \mathbf{h}\right) \equiv f(\mathbf{x})+p^{j} \cdot \operatorname{grad} p_{j}\left(\mathbf{x}^{(0)}\right) \cdot \mathbf{h}^{\downarrow}\left(\bmod p^{j+1}\right),
$$

где $\operatorname{grad} p_{j}\left(\mathbf{x}^{(0)}\right) \cdot \mathbf{h}^{\downarrow}=\sum_{i=1}^{n} \frac{\partial p_{j}}{\partial x_{i}}\left(\mathbf{x}^{(0)}\right) \cdot h_{i}$. 
Теорема 9. Любая ВКП-функция $f(\mathbf{x}) \in \mathcal{C P}_{p^{m}}(n), n \in \mathbb{N}$, сохраняет отношение сравнимости по всем делителям числа $p^{m}$.

Обобщим результаты о полиномиальных $n$-квазигруппах и биективных полиномиальных вектор-функциях, описанные в первом параграфе, на случай ВКП-функций.

По аналогии с полиномиальными функциями систему ВКП-функций $F(\mathbf{x})=\left(f_{1}(\mathbf{x}), \ldots, f_{n}(\mathbf{x})\right): \mathbb{Z}_{p^{m}}^{n} \rightarrow \mathbb{Z}_{p^{m}}^{n}, f_{i}(\mathbf{x}) \in \mathcal{C P}_{p^{m}}(n), i=\overline{1, n}, \quad$ будем называть ВКП вектор-функцией. Для ВКП вектор-функции $F(\mathbf{x})=$ $=\left(f_{1}(\mathbf{x}), \ldots, f_{n}(\mathbf{x})\right)$ будем обозначать через $F_{j}(\mathbf{x}), j=\overline{0, m-1}$, полиномиальную вектор-функцию $\left(p_{1 j}(\mathbf{x}), \ldots, p_{n j}(\mathbf{x})\right)$, где $p_{i j}(\mathbf{x}) \in \mathcal{P}_{p^{m}}(n)-j$-й координатный многочлен ВКП-функции $f_{i}(\mathbf{x}), i=\overline{1, n}$.

В предыдущем параграфе рассматривалось приведение полиномиальной функции по модулю $p^{k}, k=\overline{1, m-1}$. В силу того, что ВКП-функции сохраняют отношение сравнимости, можно определить аналогичным образом приведение ВКП вектор-функции $F(\mathbf{x})$ по модулю $p^{k}, k \in \overline{1, m-1}$, положив:

$$
F(\mathbf{x})\left(\bmod p^{k}\right)=\left(f_{1}(\mathbf{x})\left(\bmod p^{k}\right), \ldots, f_{n}(\mathbf{x})\left(\bmod p^{k}\right)\right),
$$

где $f_{i}(\mathbf{x})\left(\bmod p^{k}\right)-$ функция, получаемая путем приведения значений функции $f_{i}(\mathbf{x})$ по модулю $p^{k}$. Ясно, что определенная таким образом вектор-функция $F(\mathbf{x})\left(\bmod p^{k}\right)$ является ВКП вектор-функцией над кольцом $\mathbb{Z}_{p^{k}}$, и при $k=1$ ее можно считать вектор-функцией над полем $\mathcal{B}$. Также отметим, что ВКП вектор-функции над $\mathbb{Z}_{p^{m}}$ сохраняют отношение сравнимости по любому делителю числа $p^{m}$, т. е. из сравнения $\alpha \equiv \beta\left(\bmod p^{k}\right), k \in \overline{1, m-1}$, следует сравнение $F(\alpha) \equiv F(\beta)\left(\bmod p^{k}\right)$.

Докажем критерий того, что ВКП вектор-функция $F(\mathbf{x})=$ $=\left(f_{1}(\mathbf{x}), \ldots, f_{n}(\mathbf{x})\right): \mathbb{Z}_{p^{m}}^{n} \rightarrow \mathbb{Z}_{p^{m}}^{n}, f_{i}(\mathbf{x}) \in \mathcal{C} \mathcal{P}_{p^{m}}(n), i=\overline{1, n}$, задает биекцию.

Теорема 10. ВКП вектор-функиия $F(\mathbf{x})=\left(f_{1}(\mathbf{x}), \ldots, f_{n}(\mathbf{x})\right): \mathbb{Z}_{p^{m}}^{n} \rightarrow \mathbb{Z}_{p^{m}}^{n}$ задает биекиию тогда и только тогда, когда одновременно выполняются следуюшие условия:

1) $F_{0}(\mathbf{x})(\bmod p)$ задает биекичию на $\mathcal{B}^{n}$; 
2) для любого $j \in \overline{1, m-1}$ якобиан $\left|\mathcal{J}_{F_{j}}(\alpha)\right|(\bmod p)$ отличен от нуля при всех $\alpha \in \mathcal{B}^{n}$.

Доказательство. Необходимость. Пусть ВКП вектор-функция $F(\mathbf{x})$ задает биекцию. Тогда при любом $k \in \overline{1, m-1}$ ВКП вектор-функция $F(\mathbf{x})\left(\bmod p^{k}\right)$ над кольцом $\mathbb{Z}_{p^{k}}$ также задает биекцию. Действительно, при любом $\mathbf{y} \in \mathbb{Z}_{p^{m}}^{n}$ уравнение

$$
F(\mathbf{x})=\mathbf{y}
$$

имеет единственное решение, и при любом $k \in \overline{1, m-1}$ справедливо сравнение

$$
F(\mathbf{x}) \equiv \mathbf{y}\left(\bmod p^{k}\right) .
$$

В силу того, что $F(\mathbf{x})$ сохраняет отношение сравнимости по модулю $p^{k}$, при любом $\mathbf{y} \in \mathbb{Z}_{p^{k}}^{n}$ уравнение $F(\mathbf{x})\left(\bmod p^{k}\right)=\mathbf{y}$ имеет решение, а так как $F(\mathbf{x})\left(\bmod p^{k}\right): \mathbb{Z}_{p^{k}}^{n} \rightarrow \mathbb{Z}_{p^{k}}^{n}$, то оно единственно. Значит, $F(\mathbf{x})\left(\bmod p^{k}\right)$ задает биекцию, в частности, при $k=1$ вектор-функция $F(\mathbf{x})(\bmod p)$ биективна, что доказывает утверждение 1$)$ теоремы, поскольку $F_{0}(\mathbf{x}) \equiv F(\mathbf{x})(\bmod p)$.

Предположим, что для некоторого $j \in \overline{1, m-1}$ якобиан $\left|\mathcal{J}_{F_{j}}(\mathbf{x})\right|(\bmod p)$ равен нулю при $\mathbf{x}=\alpha \in \mathcal{B}^{n}$. Следовательно, система однородных линейных уравнений над полем $\mathcal{B}$

$$
\mathcal{J}_{F_{j}}(\alpha) \cdot \mathbf{u}^{\downarrow}={ }_{\mathcal{B}} \boldsymbol{\theta}^{\downarrow},
$$

где $\boldsymbol{\theta}^{\downarrow}-$ нулевой вектор-столбец, не определенная и потому имеет ненулевое решение $\left(\mathbf{u}^{\downarrow}\right)^{T}=\beta \in \mathcal{B}^{n}$ (т. е. $\beta \not\left(\boldsymbol{\theta}^{\downarrow}\right)^{T}(\bmod p)$ ).

Используя формулу Тейлора (теорема 8) легко показать, что для ВКП вектор-функции $F(\mathbf{x})$ справедливо сравнение

$$
F\left(\alpha+p^{j} \cdot \beta\right) \equiv F(\alpha)+p^{j} \cdot \mathcal{J}_{F_{j}}(\alpha) \cdot \beta^{\downarrow}\left(\bmod p^{j+1}\right) .
$$

Так как $\beta$ является решением системы (1), то $\mathcal{J}_{F_{j}}(\alpha) \cdot \beta^{\downarrow} \equiv \boldsymbol{\theta}^{\downarrow}(\bmod p)$, и следовательно, $p^{j} \cdot \mathcal{J}_{F_{j}}(\alpha) \cdot \beta^{\downarrow} \equiv \boldsymbol{\theta}^{\downarrow}\left(\bmod p^{j+1}\right)$, откуда получаем

$$
F\left(\alpha+p^{j} \cdot \beta\right) \equiv F(\alpha)\left(\bmod p^{j+1}\right) .
$$


В то же время $\beta \not \boldsymbol{\theta}(\bmod p)$, поэтому $\alpha+p^{j} \cdot \beta \not \alpha\left(\bmod p^{j+1}\right)$. В итоге имеем противоречие с биективностью вектор-функции $F(\mathbf{x})\left(\bmod p^{j+1}\right)$.

Достаточность. Пусть выполнены условия 1) и 2) теоремы. Докажем, что при любом $\mathbf{y}=\left(y_{1}, \ldots, y_{n}\right) \in \mathbb{Z}_{p^{m}}^{n}$ уравнение

$$
F(\mathbf{x})=\mathbf{y}
$$

имеет единственное решение. Это уравнение равносильно системе ВКПуравнений:

$$
\left\{\begin{array}{c}
f_{1}(\mathbf{x})=y_{1}, \\
\vdots \\
f_{n}(\mathbf{x})=y_{n} .
\end{array}\right.
$$

Для ее решения применим метод покоординатной линеаризации (см. [10, 14]). Приведя систему по модулю $p$, получим:

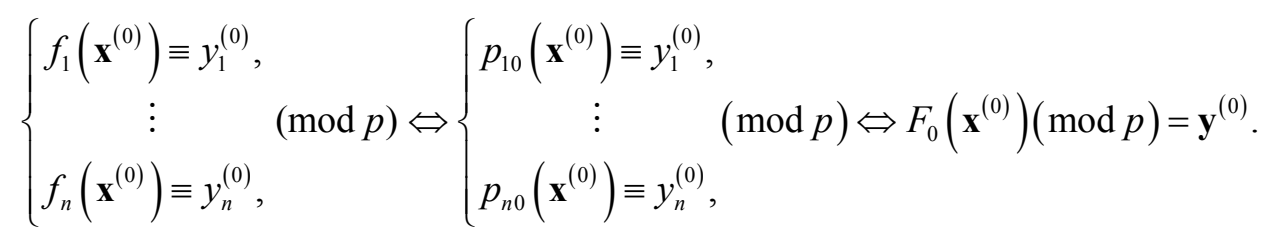

По условию теоремы уравнение $F_{0}\left(\mathbf{x}^{(0)}\right)(\bmod p)=\mathbf{y}^{(0)}$ имеет единственное решение $\mathbf{x}^{(0)}=\mathbf{c}^{(0)}$.

Пусть при $j \in \overline{1, m-1}$ найдены все значения $\mathbf{c}^{(0)}, \ldots, \mathbf{c}^{(j-1)}$ координат переменных $\mathbf{x}^{(0)}, \ldots, \mathbf{x}^{(j-1)}$ соответственно, которые удовлетворяют сравнениям вида

$$
\left\{\begin{array}{c}
f_{1}\left(\mathbf{c}^{(0)}, \ldots, \mathbf{c}^{(j-1)}\right) \equiv y_{1} \\
\vdots \\
f_{n}\left(\mathbf{c}^{(0)}, \ldots, \mathbf{c}^{(j-1)}\right) \equiv y_{n}
\end{array}\right.
$$

Приведя систему по модулю $p^{j+1}$, получим систему сравнений вида

$$
\left\{\begin{array}{c}
f_{1}\left(\mathbf{c}^{(0)}, \ldots, \mathbf{c}^{(j-1)}, \mathbf{x}^{(j)}\right) \equiv y_{1} \\
\vdots \\
f_{l}\left(\mathbf{c}^{(0)}, \ldots, \mathbf{c}^{(j-1)}, \mathbf{x}^{(j)}\right) \equiv y_{n}
\end{array}\right.
$$


Рассмотрим $i$-е уравнение полученной системы, $i \in \overline{1, n}$ :

$$
f_{i}\left(\mathbf{c}^{(0)}, \ldots, \mathbf{c}^{(j-1)}, \mathbf{x}^{(j)}\right) \equiv y_{i}\left(\bmod p^{j+1}\right) .
$$

По теореме 8 получим

$$
\begin{aligned}
& f_{i}\left(\mathbf{c}^{(0)}, \ldots, \mathbf{c}^{(j-1)}, \mathbf{x}^{(j)}\right)=f_{i}\left(\mathbf{c}^{(0)}+\ldots+p^{j-1} \mathbf{c}^{(j-1)}+p^{j} \mathbf{x}^{(j)}\right) \equiv \\
\equiv & f_{i}\left(\mathbf{c}^{(0)}, \ldots, \mathbf{c}^{(j-1)}\right)+p^{j} \cdot \operatorname{grad} p_{i j}\left(\mathbf{c}^{(0)}\right) \cdot \mathbf{x}^{(j) \downarrow} \equiv y_{i}\left(\bmod p^{j+1}\right) .
\end{aligned}
$$

В силу (2) справедливо сравнение $f_{i}\left(\mathbf{c}^{(0)}, \ldots, \mathbf{c}^{(j-1)}\right) \equiv y_{i}\left(\bmod p^{j}\right)$, а значит,

$$
f_{i}\left(\mathbf{c}^{(0)}, \ldots, \mathbf{c}^{(j-1)}\right) \equiv y_{i}^{(0)}+\ldots+p^{j-1} \cdot y_{i}^{(j-1)}+p^{j} \cdot \gamma_{j} f_{i}\left(\mathbf{c}^{(0)}, \ldots, \mathbf{c}^{(j-1)}\right)\left(\bmod p^{j+1}\right) .
$$

Отсюда имеем цепочку равносильных сравнений:

$$
\begin{gathered}
y_{i}^{(0)}+\ldots+p^{j-1} \cdot y_{i}^{(j-1)}+p^{j} \cdot \gamma_{j} f_{i}\left(\mathbf{c}^{(0)}, \ldots, \mathbf{c}^{(j-1)}\right)+ \\
+p^{j} \cdot \operatorname{grad} p_{i j}\left(\mathbf{c}^{(0)}\right) \cdot \mathbf{x}^{(j) \downarrow} \equiv y_{i}\left(\bmod p^{j+1}\right) \Leftrightarrow \\
p^{j} \cdot \gamma_{j} f_{i}\left(\mathbf{c}^{(0)}, \ldots, \mathbf{c}^{(j-1)}\right)+p^{j} \cdot \operatorname{grad} p_{i j}\left(\mathbf{c}^{(0)}\right) \cdot \mathbf{x}^{(j) \downarrow} \equiv p^{j} y_{i}^{(j)}\left(\bmod p^{j+1}\right) \\
\Leftrightarrow \operatorname{grad} p_{i j}\left(\mathbf{c}^{(0)}\right) \otimes \mathbf{x}^{(j) \downarrow}=_{\mathcal{B}} y_{i}^{(j)} \ominus \gamma_{j} f_{i}\left(\mathbf{c}^{(0)}, \ldots, \mathbf{c}^{(j-1)}\right),
\end{gathered}
$$

где операция « $\Theta »$ означает взятие противоположного элемента в аддитивной группе поля $\mathcal{B}$.

Применив таким образом указанные преобразования и рассуждения к каждому уравнению системы (3), получим равносильную ей систему линейных уравнений относительно неизвестных координат $\mathbf{x}^{(j)}$ над полем $\mathcal{B}$ (переходя к равенству $=_{\mathcal{B}}$ ):

$$
\left(\begin{array}{c}
\operatorname{grad} p_{1 j}\left(\mathbf{c}^{(0)}\right) \\
\vdots \\
\operatorname{grad} p_{n j}\left(\mathbf{c}^{(0)}\right)
\end{array}\right) \otimes \mathbf{x}^{(j) \downarrow}={ }_{\mathcal{B}}\left(\begin{array}{c}
y_{1}^{(j)} \ominus \gamma_{j} f_{1}\left(\mathbf{c}^{(0)}, \ldots, \mathbf{c}^{(j-1)}\right) \\
\vdots \\
y_{n}^{(j)} \ominus \gamma_{j} f_{n}\left(\mathbf{c}^{(0)}, \ldots, \mathbf{c}^{(j-1)}\right)
\end{array}\right) .
$$

Матрица полученной системы линейных уравнений является матрицей Якоби $\mathcal{J}_{F_{j}}\left(\mathbf{c}^{(0)}\right)(\bmod p)$ (т.е. приведенной по модулю $p$ ) полиномиальной вектор-функции $F_{j}=\left(p_{1 j}, \ldots, p_{n j}\right)$ в точке $\mathbf{c}^{(0)} \in \mathcal{B}^{n}$. Якобиан $\left|\mathcal{J}_{F_{j}}\left(\mathbf{c}^{(0)}\right)\right|(\bmod p)$ 
отличен от нуля по условию теоремы, следовательно, $\operatorname{rang} \mathcal{J}_{F_{j}}\left(\mathbf{c}^{(0)}\right)=n$. А значит, полученная система (4) имеет ровно одно решение $\mathbf{x}^{(j)}=\mathbf{c}^{(j)}$.

Таким образом, при любом $\mathbf{y}=\left(y_{1}, \ldots, y_{n}\right) \in \mathbb{Z}_{p^{m}}^{n}$ уравнение

$$
F(\mathbf{x})=\mathbf{y}
$$

имеет ровно одно решение

$$
\mathbf{c}=\sum_{j=0}^{m-1} p^{j} \cdot \mathbf{c}^{(j)} .
$$

Доказанная теорема обобщает теорему 4, которая является ее следствием, когда функции $f_{i}(\mathbf{x}), i=\overline{1, n}$, полиномиальны.

При $n=1$ из теоремы следует критерий биективности (подстановочности) ВКП-функции от одной переменной, который является обобщением теоремы 1. Такие ВКП-функции будем называть подстановочными ВКПфункциями.

Следствие. Функция $f(x) \in \mathcal{C P}_{p^{m}}(1)$ с координатными многочленами $p_{0}(x), \ldots, p_{m-1}(x)$ задает биективное преобразование (подстановку) кольца $\mathbb{Z}_{p^{m}}$ тогда и только тогда, когда одновременно выполняются следуюшие условия:

1) $p_{0}(x)(\bmod p)$ задает биективное преобразование поля $\mathcal{B}$;

2) для любого $j \in \overline{1, m-1}$ многочлен $p_{j}^{\prime}(x)(\bmod p)$ не имеет корней в поле $\mathcal{B}$.

Сформулируем еще одно следствие из доказанной теоремы, которое позволяет явным образом строить биективные ВКП вектор-функции.

Следствие. Если полиномиальные вектор-функциии $G_{0}(\mathbf{x}), \ldots, G_{m-1}(\mathbf{x})$ над кольиом $\mathbb{Z}_{p^{m}}$ задают биекцию, то ВКП вектор-функция $F(\mathbf{x})$, для которой

$$
F_{j}(\mathbf{x})=G_{j}(\mathbf{x}), j=\overline{0, m-1},
$$

также задает биекиию.

Приведенное следствие позволяет строить биективные ВКП векторфункции, используя биективные полиномиальные вектор-функции. Кроме 
того, используя теорему 7, можно получать при этом не полиномиальные ВКП вектор-функции и подстановочные ВКП-функции.

Пример 3. Рассмотрим ВКП-функцию $f(x)$ от одной переменной над $\mathbb{Z}_{27}$ с координатными многочленами:

$$
\begin{gathered}
p_{0}(x)=x+1, \\
p_{1}(x)=2 x, \\
p_{2}(x)=x+2 .
\end{gathered}
$$

Формальные производные многочленов $p_{1}(x)$ и $p_{2}(x)$ равны соответственно:

$$
p_{1}^{\prime}(x)=2, p_{2}^{\prime}(x)=1 \text {. }
$$

Следовательно, по теореме 7 функция $f(x)$ не полиномиальна. Очевидно, что при этом каждый координатный многочлен задает биекцию кольца, а значит, и $f(x)$ задает биекцию. В нижней строке таблицы 2 представлены значения функции $f(x)$.

Таблица 2

\begin{tabular}{|l|l|l|l|l|l|l|c|c|c|c|c|c|c|c|c|c|c|c|c|c|c|c|c|c|c|c|}
\hline 0 & 1 & 2 & 3 & 4 & 5 & 6 & 7 & 8 & 9 & 10 & 11 & 12 & 13 & 14 & 15 & 16 & 17 & 18 & 19 & 20 & 21 & 22 & 23 & 24 & 25 & 26 \\
\hline $\mathbf{1}$ & $\mathbf{2}$ & $\mathbf{3}$ & $\mathbf{7}$ & $\mathbf{8}$ & $\mathbf{0}$ & $\mathbf{4}$ & $\mathbf{1 4}$ & $\mathbf{1 5}$ & $\mathbf{1 0}$ & $\mathbf{1 1}$ & $\mathbf{1 2}$ & $\mathbf{1 6}$ & $\mathbf{1 7}$ & $\mathbf{9}$ & $\mathbf{1 3}$ & $\mathbf{2 3}$ & $\mathbf{2 4}$ & $\mathbf{1 9}$ & $\mathbf{2 0}$ & $\mathbf{2 1}$ & $\mathbf{2 5}$ & $\mathbf{2 6}$ & $\mathbf{1 8}$ & $\mathbf{2 2}$ & $\mathbf{5}$ & $\mathbf{6}$ \\
\hline
\end{tabular}

В то же время можно строить биективные ВКП вектор-функции, используя полиномиальные вектор-функции, которые биекций не задают.

Пример 4. Рассмотрим ВКП-функцию $f(x)$ над $\mathbb{Z}_{27}$ с координатными многочленами:

$$
\begin{gathered}
p_{0}(x)=x+1 \\
p_{1}(x)=8 x^{3}+7 x+3 \\
p_{2}(x)=x^{3}+2 x
\end{gathered}
$$

Аналогично предыдущему примеру имеем: $p_{0}(x)$ задает биекцию по модулю 3 и при этом

$$
\begin{gathered}
p_{1}^{\prime}(x)=24 x^{2}+7 \equiv 1(\bmod 3), \\
p_{2}^{\prime}(x)=3 x^{2}+2 \equiv 2(\bmod 3) .
\end{gathered}
$$


М. В. Заец

Отсюда следует, что $f(x)$ не полиномиальна. По теореме 10 функция $f(x)$ задает биекцию на кольце $\mathbb{Z}_{27}$. Легко проверить, что в то же время $p_{1}(x)$ и $p_{2}(x)$ не задают биекции на кольце $\mathbb{Z}_{27}$, поскольку:

$$
\begin{aligned}
& p_{1}(x) \equiv 2 x+x \equiv 0(\bmod 3), \\
& p_{2}(x) \equiv x+2 x \equiv 0(\bmod 3) .
\end{aligned}
$$

В нижней строке таблицы 3 представлены значения данной функции.

Таблица 3

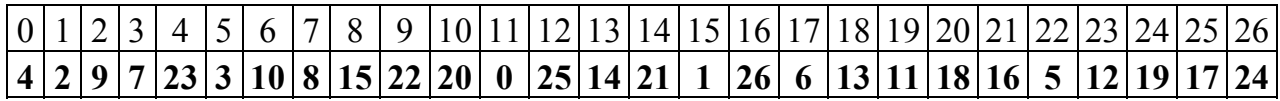

Докажем далее утверждения об $n$-квазигруппах для более общего случая полиномиальных $n$-квазигрупп. А именно, сформулируем критерий того, что ВКП-функция $f(\mathbf{x}) \in \mathcal{C P}_{p^{m}}(n)$ задает $n$-квазигруппу на кольце $\mathbb{Z}_{p^{m}}$. Такие $n$-квазигруппы будем по аналогии назвать ВКП $n$-квазигруппами.

Теорема 11. Функиия $f(\mathbf{x}) \in \mathcal{C P}_{p^{m}}(n)$ с координатными многочленами $p_{0}(\mathbf{x}), \ldots, p_{m-1}(\mathbf{x})$ задает n-квазигруппу на кольще $\mathbb{Z}_{p^{m}}$ тогда и только тогда, когда выполняются следующие условия:

1) $p_{0}(\mathbf{x})(\bmod p)$ задает n-квазигруппу на $\mathcal{B}$;

2) для любого $j \in \overline{1, m-1}$ вектор-функиия $\operatorname{grad} p_{j}(\alpha)(\bmod p)$ не содержит нулевых координат при любом $\alpha \in \mathcal{B}^{n}$.

Доказательство. При $n=1$ теорема 11 есть следствие теоремы 10 . Пусть $f(\mathbf{x}) \in \mathcal{C P}_{p^{m}}(n), n>1$, докажем справедливость теоремы с помощью последовательности равносильных переходов.

Для вектора $\alpha=\left(a_{1}, \ldots, a_{n-1}\right) \in \mathbb{Z}_{p^{m}}^{n-1}$ и набора $\imath=\left(i_{1}, \ldots, i_{n-1}\right): i_{1}<\ldots<$ $<i_{n-1}, i_{s} \in \overline{1, n}, s=\overline{1, n-1}$, обозначим через $g_{l}^{\alpha}(x)=f_{l}^{\alpha}(\mathbf{x})$ подфункцию функции $f(\mathbf{x})$, получаемую фиксацией переменных: $x_{i_{s}}=a_{s}, s=\overline{1, n-1}$. Функция $g_{\imath}^{\alpha}(x)$ зависит от одной нефиксированной переменной функции $f(\mathbf{x})$ и является ВКП-функцией с координатными многочленами $\left(p_{0}\right)_{t}^{\alpha}(\mathbf{x}), \ldots$, $\left(p_{m-1}\right)_{\imath}^{\alpha}(\mathbf{x})$. 
Функция $f(\mathbf{x}) \in \mathcal{C} \mathcal{P}_{p^{m}}(n)$ задает $n$-квазигруппу тогда и только тогда, когда при любом векторе $\alpha \in \mathbb{Z}_{p^{m}}^{n-1}$ и любом наборе номеров переменных $\imath=\left(i_{1}, \ldots, i_{n-1}\right)$ ВКП-функция $g_{l}^{\alpha}(x)$ является биекцией, а это выполняется тогда и только тогда, когда для нее справедливы условия первого следствия теоремы 3.1, т. е.:

1) $g_{\imath}^{\alpha}(x) \equiv\left(p_{0}\right)_{\imath}^{\alpha}(\mathbf{x}) \equiv\left(p_{0}\right)_{t}^{\alpha^{(0)}}(\mathbf{x})(\bmod p)$ задает биекцию поля $\mathcal{B}$;

2) для любого $j \in \overline{1, m-1}: \quad\left(\left(p_{j}\right)_{t}^{\alpha}(\mathbf{x})\right)^{\prime} \equiv\left(p_{j}^{\prime}\right)_{\imath}^{\alpha^{(0)}}(\mathbf{x})(\bmod p)$ не имеет корней в поле $\mathcal{B}$.

В силу произвольности выбора $\alpha \in \mathbb{Z}_{p^{m}}^{n-1}$ и набора номеров переменных $\imath=\left(i_{1}, \ldots, i_{n-1}\right)$ сказанное можно записать в следующей эквивалентной форме:

1) для любых $\alpha \in \mathcal{B}^{n-1} \quad$ и набора $\quad l=\left(i_{1}, \ldots, i_{n-1}\right)$ функция $\left(p_{0}\right)_{t}^{\alpha}(\mathbf{x})(\bmod p)$ задает биекцию поля $\mathcal{B}$, а это означает, что $p_{0}(\mathbf{x})(\bmod p)$ задает $n$-квазигруппу на поле $\mathcal{B}$;

2) для любых $\alpha \in \mathcal{B}^{n}$ и $j \in \overline{1, m-1}$ значение $\frac{\partial p_{j}}{\partial x_{s}}(\alpha) \neq 0(\bmod p), s=\overline{1, n}$, а это равносильно тому, что $\operatorname{grad} p_{j}(\alpha)(\bmod p)$ не содержит нулевых координат.

Следствие. Если полиномиальньее функции $p_{0}(\mathbf{x}), \ldots, p_{m-1}(\mathbf{x}) \in \mathcal{P}_{p^{m}}(n)$ задают п-квазигруппу, то ВКП-функиия $f(\mathbf{x}) \in \mathcal{C P}_{p^{m}}(n)$ с данньлми координатными многочленами также задает п-квазигруппу.

Доказанная теорема действительно обобщает теорему 3 поскольку, используя ее, можно строить неполиномиальные $n$-квазигруппы. В частности, при $n=2$ и $m \geq 3$ можно строить латинские квадраты, не имеющие полиномиального задания. Кроме того, отметим, что приведенное в следствии условие не является необходимым, как показывает следующий пример.

Пример 5. Рассмотрим ВКП-функцию $f(x, y) \in \mathcal{C P}_{27}(2)$ с координатными многочленами:

$$
\begin{gathered}
p_{0}(x, y)=x+y \\
p_{1}(x, y)=x^{3}+5 x+6 x y+2 y+y^{3} \\
p_{2}(x, y)=2 x^{3}+x+3 x y+y+2 y^{3}
\end{gathered}
$$


Проверим, что координатные многочлены удовлетворяют условию теоремы 11. Многочлен

$$
p_{0}(x, y) \equiv x+y(\bmod 3)
$$

задает 2-квазигруппу (латинский квадрат) на $\mathcal{B}$. И в то же время:

$$
\begin{gathered}
\operatorname{grad} p_{1}(x, y)=(5+6 y, 2+6 x) \equiv(2,2)(\bmod 3), \\
\operatorname{grad} p_{2}(x, y)=(1+3 y, 1+3 x) \equiv(1,1)(\bmod 3) .
\end{gathered}
$$

Поэтому $f(x, y)$ по теореме 11 задает 2-квазигруппу на $\mathbb{Z}_{27}$. При этом $f(x, y)$ не полиномиальна по теореме 7 , и координатные многочлены $p_{1}(x, y), p_{2}(x, y)$ не задают 2-квазигруппы, поскольку

$$
\begin{aligned}
& p_{1}(x, y) \equiv 0(\bmod 3), \\
& p_{2}(x, y) \equiv 0(\bmod 3) .
\end{aligned}
$$

Приведем общую конструкцию построения ВКП $n$-квазигрупп с помощью аффинных функций над произвольным примарным кольцом $\mathbb{Z}_{p^{m}}$. Пусть

$$
p_{j}(\mathbf{x})=a_{j 1} x_{1}+\ldots+a_{j n} x_{n}+a_{j}, a_{j i}, a_{j} \in \mathbb{Z}_{p^{m}}, i=\overline{1, n}, j=\overline{0, m-1} .
$$

Легко проверить, что $p_{0}(\mathbf{x})(\bmod p)$ задает $n$-квазигруппу на $\mathcal{B}$ тогда и только тогда, когда все коэффициенты $a_{0 i}, i=\overline{1, n}$, обратимы, это равносильно тому, что $p_{0}(\mathbf{x})$ задает $n$-квазигруппу на $\mathbb{Z}_{p^{m}}$. Градиент многочлена $p_{j}(\mathbf{x})(\bmod p), j=\overline{1, m-1}$, равен

$$
\operatorname{grad} p_{j}(\mathbf{x})=\left(a_{j 1}, \ldots, a_{j n}\right) \equiv\left(a_{j 1}^{(0)}, \ldots, a_{j n}^{(0)}\right)(\bmod p)
$$

и не содержит нулевых координат тогда и только тогда, когда коэффициенты $a_{j i}, i=\overline{1, n}$, обратимы. Отсюда получаем критерий того, что ВКП-функция $f(\mathbf{x})$ с аффинными координатными многочленами задает $n$-квазигруппу: $f(\mathbf{x})$ задает $n$-квазигруппу тогда и только тогда, когда ее координатные многочлены задают $n$-квазигруппу, и это равносильно тому, что $a_{j i} \in \mathbb{Z}_{p^{m}}^{*}, i=\overline{1, n}, j=\overline{0, m-1}$.

Изучим далее некоторые свойства ВКП $n$-квазигрупп. Напомним для этого некоторые сведения об $n$-квазигруппах из [9]. 
Пусть $(Q, f)$ - произвольная $n$-квазигруппа, тогда в равенстве

$$
f\left(x_{1}, \ldots, x_{n}\right)=x_{n+1}
$$

любые $n$ элементов однозначно определяют $(n+1)$-й, в частности, при любом $i \in \overline{1, n}$ набор элементов $x_{1}, \ldots, x_{i-1}, x_{n+1}, x_{i+1}, \ldots, x_{n}$ однозначно определяет элемент $x_{i}$ из (5). Это позволяет определить $n$-арную операцию ${ }^{\pi_{i}} f$ равенством

$$
\pi_{i} f\left(x_{1}, \ldots, x_{i-1}, x_{n+1}, x_{i+1}, \ldots, x_{n}\right)=x_{i} .
$$

Определение 6. Пусть $(Q, f)-n$-квазигруппа, $i \in \overline{1, n}$. Операция ${ }_{i} f$, определяемая равенством (6), эквивалентным равенству (5), называется $i$-й обратной операцией $\kappa f$.

Известно, что операция $\pi_{i} f$ также задает $n$-квазигруппу на $Q$ (см. [9]). Данную конструкцию можно обобщить следующим образом. Пусть $\sigma \in S_{n+1}$ и $(Q, f)-n$-квазигруппа; определим операцию ${ }^{\sigma} f$ равенством:

$$
{ }^{\sigma} f\left(x_{\sigma(1)}, \ldots, x_{\sigma(n)}\right)=x_{\sigma(n+1)} \Leftrightarrow f\left(x_{1}, \ldots, x_{n}\right)=x_{n+1} .
$$

Полученнная операция определена корректно и также является $n$-квазигрупппой (см. [9]).

Определение 7. Пусть $(Q, f)-n$-квазигруппа и подстановка $\sigma \in S_{n+1}$. Операция ${ }^{\sigma} f$, определяемая равенством

$$
\sigma f\left(x_{\sigma(1)}, \ldots, x_{\sigma(n)}\right)=x_{\sigma(n+1)},
$$

эквивалентным равенству (5), называется $\sigma$-парастрофом операщии $f$, или просто парастрофом. При этом парастроф с условием $\sigma(n+1)=n+1$, называется главным.

В частности, из определения 7 следует, что $i$-я обратная операция $n$-квазигруппы является ее $\pi_{i}$-парастрофом, где $\pi_{i}=(i, n+1)$ - транспозиция. Также непосредственной проверкой доказывается, что любой парастроф $n$-квазигруппы задает $n$-квазигруппу. В дальнейшем нам понадобится теорема о связи парастрофов $n$-квазигруппы и обратных операций.

Теорема 12 ([9]). Всякий парастроф п-квазигруппь $(Q, f)$ может быть получен последовательным применением обратных операций. 
М. В. Заец

Теперь перейдем к рассмотрению $n$-квазигрупп на кольце $\mathbb{Z}_{p^{m}}$. Докажем, что если функция $f(\mathbf{x})$ сохраняет отношение сравнимости по любому делителю числа $p^{m}$, то этим свойством обладает и ${ }^{\pi_{i}} f$. Сформулируем следующий результат.

Лемма 13. Если функиия $f(x)$ сохраняет отношение сравнимости по любомуделителю $p^{m}$ u $f(x)$ задает биекиию на кольще $\mathbb{Z}_{p^{m}}$, то при любом $k \in \overline{1, m-1}$ функиия $f(x)\left(\bmod p^{k}\right)$ также задает биекцию на кольие $\mathbb{Z}_{p^{k}}$.

Доказательство. Доказательство леммы проводится аналогично доказательству необходимости в теореме 10 .

Следствие. Если функция $f(\mathbf{x})$ сохраняет отнотение сравнимости по любому делителю $p^{m}$ u $f(\mathbf{x})$ задает п-квазигруппу на кольце $\mathbb{Z}_{p^{m}}$, то при любом $k \in \overline{1, m-1}$ функиия $f(\mathbf{x})\left(\bmod p^{k}\right)$ задает n-квазигруппу на кольие $\mathbb{Z}_{p^{k}}$.

Пусть $f(\mathbf{x})$ сохраняет отношение сравнимости по любому делителю числа $p^{m}$ и $f(\mathbf{x})$ задает $n$-квазигруппу на $\mathbb{Z}_{p^{m}}, n>1$. Рассмотрим эпиморфизм абелевых групп $\tau_{k}:\left(\mathbb{Z}_{p^{m}},+\right) \rightarrow\left(\mathbb{Z}_{p^{k}},+\right)$, действующий по правилу $\tau_{k}(a)=a\left(\bmod p^{k}\right), k \in \overline{1, m-1}$. Тогда $\tau_{k}$ также является эпиморфизмом $n$-квазигрупп $\left(\mathbb{Z}_{p^{m}}, f\right)$ и $\left(\mathbb{Z}_{p^{k}}, \hat{f}\right)$, где $\hat{f}(\mathbf{x})$ - приведение $f(\mathbf{x})$ по модулю $p^{k}$. Следовательно, $\tau_{k}$ задает на $n$-квазигруппе $\left(\mathbb{Z}_{p^{m}}, f\right)$ конгруэнцию $\rho_{k}$ :

$$
a \rho_{k} b \Leftrightarrow \tau_{k}(a)=\tau_{k}(b),
$$

(другими словами, $\rho_{k}$ - отношение сравнимости по модулю $p^{k}$ ), которую по аналогии с 2-квазигруппами (см. [8]) можно назвать нормальной. То есть конгруэнция $n$-квазигруппы $\left(\mathbb{Z}_{p^{m}}, f\right)$ нормальна, если обладает свойством: для $\quad$ любых $\alpha=\left(a_{1}, \ldots, a_{n-1}\right) \in \mathbb{Z}_{p^{m}}^{n-1} \quad$ и $\quad$ набора $\quad l=\left(i_{1}, \ldots, i_{n-1}\right): i_{1}<\ldots<i_{n-1}$, $i_{s} \in \overline{1, n}, s=\overline{1, n-1}$, из условия

$$
f_{l}^{\alpha}(a) \equiv f_{l}^{\alpha}(b)\left(\bmod p^{k}\right)
$$


следует, что

$$
a \equiv b\left(\bmod p^{k}\right)
$$

Действительно, если $f_{l}^{\alpha}(a) \equiv f_{l}^{\alpha}(b)\left(\bmod p^{k}\right)$, то $\tau_{k}\left(f_{l}^{\alpha}(a)\right)=\tau_{k}\left(f_{l}^{\alpha}(b)\right)$ и $\hat{f}_{t}^{\tau_{k}^{*}(\alpha)}\left(\tau_{k}(a)\right)=\hat{f}_{l}^{\tau_{k}^{*}(\alpha)}\left(\tau_{k}(b)\right)$, где $\tau_{k}^{*}(\alpha)=\left(\tau_{k}\left(a_{1}\right), \ldots, \tau_{k}\left(a_{n-1}\right)\right)$ и $\hat{f}(\mathbf{x})$ функция, равная $f(\mathbf{x})\left(\bmod p^{k}\right)$. Отсюда в силу того, что $\hat{f}_{t}^{\tau_{k}^{*}(\alpha)}-$ биекция $\mathbb{Z}_{p^{k}}$, следует равенство $\tau_{k}(a)=\tau_{k}(b)$, а это означает $a \equiv b\left(\bmod p^{k}\right)$.

Утверждение 14. Если функция $f(\mathbf{x})$ сохраняет отночение сравнимости по любому делителю числа $p^{m}$ u $f(\mathbf{x})$ задает $n$-квазигруппу на $\mathbb{Z}_{p^{m}}$, то любая ей обратная операцчия ${ }^{\pi_{i}} f, i=\overline{1, n}$, также обладает данным свойством.

Доказательство. Если $n=1$, то утверждение непосредственно следует из леммы 13. Пусть $n>1$. При $k \in \overline{1, m-1}$, докажем, что ${ }^{\pi_{i}} f(\mathbf{x})$ сохраняет отношение сравнимости по модулю $p^{k}$. Пусть $a_{s} \equiv b_{s}\left(\bmod p^{k}\right)$, $s=\overline{1, n+1}, s \neq i, \quad a_{i}={ }^{\pi_{i}} f\left(a_{1}, \ldots, a_{n+1}, \ldots, a_{n}\right), \quad b_{i}={ }^{\pi_{i}} f\left(b_{1}, \ldots, b_{n+1}, \ldots, b_{n}\right) ; \quad$ надо показать, что $a_{i} \equiv b_{i}\left(\bmod p^{k}\right)$. Согласно равенствам (5) и (6) имеем:

$$
f\left(a_{1}, \ldots, a_{i}, \ldots, a_{n}\right)=a_{n+1} \equiv_{p^{k}} b_{n+1}=f\left(b_{1}, \ldots, b_{i}, \ldots, b_{n}\right) .
$$

Так как $f(\mathbf{x})$ сохраняет отношение сравнимости по модулю $p^{k}$, то

$$
f\left(b_{1}, \ldots, b_{i}, \ldots, b_{n}\right) \equiv f\left(a_{1}, \ldots, b_{i}, \ldots, a_{n}\right)\left(\bmod p^{k}\right),
$$

откуда следует сравнение

$$
f\left(a_{1}, \ldots, a_{i}, \ldots, a_{n}\right) \equiv f\left(a_{1}, \ldots, b_{i}, \ldots, a_{n}\right)\left(\bmod p^{k}\right) .
$$

Оно равносильно сравнению $f_{l}^{\alpha}\left(a_{i}\right) \equiv f_{l}^{\alpha}\left(b_{i}\right)\left(\bmod p^{k}\right), \quad$ где $\alpha=\left(a_{1}, \ldots a_{i-1}, a_{i+1}, \ldots, a_{n}\right)$ и $\imath=(1, \ldots, i-1, i+1, \ldots, n)$. Отсюда в силу нормальности конгруэнции $\rho_{k}$ вытекает сравнение $a_{i} \equiv b_{i}\left(\bmod p^{k}\right)$.

Следующий результат получается применением доказанного утверждения к теореме 12. 
М. В. Заец

Следствие. Если $f(\mathbf{x})$ сохраняет отномение сравнимости по любому делителю $p^{m}$ и $f(\mathbf{x})$ задает n-квазигруппу на $\mathbb{Z}_{p^{m}}$, то любой ее парастроф ${ }^{\sigma} f$ также обладает данным свойством.

Из утверждения 14 вытекает, что если ВКП-функция $f(\mathbf{x}) \in \mathcal{C P}_{p^{m}}(n)$ задает $n$-квазигруппу на кольце $\mathbb{Z}_{p^{m}}$, то любая ее $i$-я обратная операция и любой ее парастроф сохраняют отношение сравнимости по любому делителю числа $p^{m}$. Интересным в таком случае представляется вопрос о том, является ли $i$-я обратная операция ВКП-функцией. В некотором смысле ответ на него будет получен далее. Для этого нам потребуется обобщить класс ВКП-функций следующей конструкцией (см. [15]).

Определение 8. Функцию $f(\mathbf{x})$ назовем квази-вариаџионно-координатно полиномиальной (или квази-ВКП-функцией), если выполнены условия:

1. $\gamma_{0} f(\mathbf{x})=\gamma_{0} f\left(\mathbf{x}^{(0)}\right)=g_{0}\left(\mathbf{x}^{(0)}\right), g_{0}: \mathcal{B}^{n} \rightarrow \mathcal{B} ;$

2. для любого $j \in \overline{1, m-1}: \gamma_{j} f(\mathbf{x})=\gamma_{j} f\left(\mathbf{x}^{(0)}, \ldots, \mathbf{x}^{(j)}\right)$ и существуют такие функции $g_{j i}: \mathcal{B}^{n} \rightarrow \mathcal{B}, g_{j}: \mathcal{B}^{\text {jn }} \rightarrow \mathcal{B}, i=\overline{1, n}$, над полем $\mathcal{B}$, что справедливо равенство

$$
\gamma_{j} f\left(\mathbf{x}^{(0)}, \ldots, \mathbf{x}^{(j)}\right)=\sum_{i=1}^{n} g_{j i}\left(\mathbf{x}^{(0)}\right) \otimes x_{i}^{(j)} \oplus g_{j}\left(\mathbf{x}^{(0)}, \ldots, \mathbf{x}^{(j-1)}\right) .
$$

Класс всех квази-ВКП-функций от $n$ переменных над кольцом $\mathbb{Z}_{p^{m}}$ обозначим $\mathcal{Q C P}_{p^{m}}(n)$. Можно доказать, что справедливо включение

$$
\mathcal{C} \mathcal{P}_{p^{m}}(n) \subseteq \mathcal{Q C P}_{p^{m}}(n)
$$

При этом в некоторых случаях включение может обращаться в равенство. А именно при $m=2$ рассматриваемые классы функций совпадают ([15]).

Оказывается, что для ВКП $n$-квазигруппы ее обратная операция является по крайней мере квази-ВКП-функцией. Этот результат содержится в следующей теореме.

Теорема 15. Если $f(\mathbf{x}) \in \mathcal{C P}_{p^{m}}(n), f(\mathbf{x})$ задает п-квазигруппу на $\mathbb{Z}_{p^{m}}$, $i \in \overline{1, n}$, mo $^{\pi_{i}} f \in \mathcal{Q C P}_{p^{m}}(n)$. 
Доказательство. Без ограничения общности доказательство будем проводить для 1-й обратной операции. Пусть $f(\mathbf{x}) \in \mathcal{C P}_{p^{m}}(n)$ с координатными многочленами $p_{0}(\mathbf{x}), \ldots, p_{m-1}(\mathbf{x})$ задает $n$-квазигруппу на $\mathbb{Z}_{p^{m}}$. Найдем выражение для координатных функций 1-й обратной операции ${ }^{\pi_{1}} f(\mathbf{u}), \mathbf{u}=\left(x_{n+1}, x_{2}, \ldots, x_{n}\right)$. Для этого рассмотрим координатные функции ВКП-функции $f(\mathbf{x})$.

Имеем:

$$
\begin{gathered}
f\left(x_{1}, \ldots, x_{n}\right)=x_{n+1} \Leftrightarrow \\
x_{n+1}^{(0)}=g_{0}\left(\mathbf{x}^{(0)}\right), \\
x_{n+1}^{(j)}=\sum_{i=1}^{n} g_{j i}\left(\mathbf{x}^{(0)}\right) \otimes x_{i}^{(j)} \oplus g_{j}\left(\mathbf{x}^{(0)}, \ldots, \mathbf{x}^{(j-1)}\right), j=\overline{1, m-1} .
\end{gathered}
$$

Тогда $p_{0}(\mathbf{x}) \equiv p_{0}\left(\mathbf{x}^{(0)}\right) \equiv g_{0}\left(\mathbf{x}^{(0)}\right)(\bmod p)$, а поскольку $p_{0}(\mathbf{x})(\bmod p)$ по теореме 11 задает $n$-квазигруппу на $\mathcal{B}$, то ее задает на $\mathcal{B}$ и $g_{0}\left(\mathbf{x}^{(0)}\right)$. Поэтому $x_{1}^{(0)}={ }^{\pi_{1}} g_{0}\left(\mathbf{u}^{(0)}\right)$.

Нетрудно показать, что при $j=\overline{1, m-1}, i=\overline{1, n}$ справедливо сравнение

$$
\frac{\partial p_{j}}{\partial x_{i}}(\mathbf{x}) \equiv \frac{\partial p_{j}}{\partial x_{i}}\left(\mathbf{x}^{(0)}\right) \equiv g_{j i}\left(\mathbf{x}^{(0)}\right)(\bmod p) .
$$

А значит, по теореме 11 функции $g_{j i}\left(\mathbf{x}^{(0)}\right)$ не обращаются в нуль ни при каком $\mathbf{x}^{(0)} \in \mathcal{B}^{n}, j=\overline{1, m-1}, i=\overline{1, n}$. В частности, это верно при $i=1$. Следовательно, существуют такие полиномиальные функции $v_{j 1}\left(\mathbf{x}^{(0)}\right), j \in \overline{1, m-1}$, что для всех $\mathbf{x}^{(0)} \in \mathcal{B}^{n}$ выполнено равенство в поле $\mathcal{B}$ :

$$
v_{j 1}\left(\mathbf{x}^{(0)}\right) \otimes g_{j 1}\left(\mathbf{x}^{(0)}\right)={ }_{\mathcal{B}} 1, j=\overline{1, m-1},
$$

т. е. $v_{j 1}\left(\mathbf{x}^{(0)}\right)=\left(g_{j 1}\left(\mathbf{x}^{(0)}\right)\right)^{-1}$. Умножив обе части равенства (8) на $v_{j 1}\left(\mathbf{x}^{(0)}\right)$, получим:

$$
\begin{gathered}
x_{n+1}^{(j)} \otimes v_{j 1}\left(\mathbf{x}^{(0)}\right)=x_{1}^{(j)} \oplus \\
\oplus\left(\sum_{i=2}^{n} g_{j i}\left(\mathbf{x}^{(0)}\right) \otimes x_{i}^{(j)} \oplus g_{j}\left(\mathbf{x}^{(0)}, \ldots, \mathbf{x}^{(j-1)}\right)\right) \otimes v_{j 1}\left(\mathbf{x}^{(0)}\right) \Leftrightarrow
\end{gathered}
$$




$$
\begin{gathered}
\Leftrightarrow x_{1}^{(j)}=x_{n+1}^{(j)} \otimes v_{j 1}\left(\mathbf{x}^{(0)}\right) \ominus \sum_{i=2}^{n} g_{j i}\left(\mathbf{x}^{(0)}\right) \otimes v_{j 1}\left(\mathbf{x}^{(0)}\right) \otimes x_{i}^{(j)} \ominus \\
\ominus g_{j}\left(\mathbf{x}^{(0)}, \ldots, \mathbf{x}^{(j-1)}\right) \otimes v_{j 1}\left(\mathbf{x}^{(0)}\right) .
\end{gathered}
$$

Воспользовавшись тем, что $x_{1}^{(0)}={ }^{\pi_{1}} g_{0}\left(\mathbf{u}^{(0)}\right)$, придем к цепочке равенств:

$$
\begin{gathered}
v_{j 1}\left(\mathbf{x}^{(0)}\right)=v_{j 1}\left(x_{1}^{(0)}, x_{2}^{(0)}, \ldots, x_{n}^{(0)}\right)=v_{j 1}\left({ }^{\pi_{1}} g_{0}\left(\mathbf{u}^{(0)}\right), x_{2}^{(0)}, \ldots, x_{n}^{(0)}\right)= \\
=h_{j, n+1}\left(\mathbf{u}^{(0)}\right) .
\end{gathered}
$$

Аналогично:

$$
\ominus g_{j i}\left(\mathbf{x}^{(0)}\right) \otimes v_{j 1}\left(\mathbf{x}^{(0)}\right)=h_{j i}\left(\mathbf{u}^{(0)}\right) .
$$

Поскольку 1-я обратная операция ${ }^{\pi_{1}} f(\mathbf{u})$ по утверждению 14 сохраняет отношение сравнимости, то

$$
x_{1}^{(j)}=\gamma_{j}{ }^{\pi_{1}} f(\mathbf{u})=\gamma_{j}{ }^{\pi_{1}} f\left(\mathbf{u}^{(0)}, \ldots, \mathbf{u}^{(j)}\right)=a_{j}\left(\mathbf{u}^{(0)}, \ldots, \mathbf{u}^{(j)}\right), j=\overline{1, m-1} .
$$

И тогда можно записать:

$$
\begin{gathered}
\ominus g_{j}\left(\mathbf{x}^{(0)}, \ldots, \mathbf{x}^{(j-1)}\right) \otimes v_{j 1}\left(\mathbf{x}^{(0)}\right)= \\
=\ominus g_{j}\left({ }^{\pi_{1}} g_{0}\left(\mathbf{u}^{(0)}\right), \ldots, a_{1}\left(\mathbf{u}^{(0)}, \mathbf{u}^{(1)}\right), \ldots, a_{j-1}\left(\mathbf{u}^{(0)}, \ldots, \mathbf{u}^{(j-1)}\right), \ldots, x_{n}^{(j-1)}\right) \otimes \\
\otimes h_{j, n+1}\left(\mathbf{u}^{(0)}\right)=h_{j}\left(\mathbf{u}^{(0)}, \ldots, \mathbf{u}^{(j-1)}\right) .
\end{gathered}
$$

Подставляя все полученное в равенство (9), находим:

$$
x_{1}^{(j)}=\sum_{i=2}^{n+1} h_{j i}\left(\mathbf{u}^{(0)}\right) \otimes x_{i}^{(j)} \oplus h_{j}\left(\mathbf{u}^{(0)}, \ldots, \mathbf{u}^{(j-1)}\right), \quad j=\overline{1, m-1} .
$$

Таким образом, все координатные функции 1-й обратной операции ${ }_{1} f(\mathbf{u})$ имеют вид, указанный в определении 8. А значит, ${ }^{\pi_{1}} f \in \mathcal{Q C P}_{p^{m}}(n)$.

Ряд доказанных выше утверждений о свойствах ВКП-функций можно обобщить на класс квази-ВКП-функций. Так, например, теорему 11 с незначительными изменениями можно сформулировать и для квази-ВКПфункций $f(\mathbf{x}) \in \mathcal{Q C P}_{p^{m}}(n)$. Для этого нужно ввести определение $j$-го координатного градиента квази-ВКП-функции:

$$
\operatorname{grad}_{j} f(\alpha)=\left(g_{j 1}(\alpha), \ldots, g_{j n}(\alpha)\right),
$$


и потребовать, чтобы:

1) функция $g_{0}\left(\mathbf{x}^{(0)}\right)$ задавала $n$-квазигруппу на $\mathcal{B}$,

2) для любого $j \in \overline{1, m-1}$ градиент $\operatorname{grad}_{j} f(\alpha)$ не содержал нулевых координат при любом $\alpha \in \mathcal{B}^{n}$.

В п. 1, 2 получаем обобщение теоремы 11, так как в случае ВКП-функций $g_{0}\left(\mathbf{x}^{(0)}\right) \equiv p_{0}(\mathbf{x})(\bmod p)$ и вектор $\operatorname{grad}_{j} f(\alpha) \equiv \operatorname{grad} p_{j}(\alpha)(\bmod p)$. Поэтому в такой формулировке получаем обобщение теоремы 11. Доказательство данного факта аналогично доказательству теорем 10 и 11.

Используя этот результат, можно также обобщить теорему 15 на случай квази-ВКП-функций.

Теорема 16. Если $f(\mathbf{x}) \in \mathcal{Q C P}_{p^{m}}(n), f(\mathbf{x})$ задает $n$-квазигруппу на $\mathbb{Z}_{p^{m}}$, $i \in \overline{1, n}, m o{ }^{\pi_{i}} f \in \mathcal{Q C P}_{p^{m}}(n)$.

Из теорем 16 и 12 непосредственно вытекает следующее утверждение.

Следствие. Если $f(\mathbf{x}) \in \mathcal{Q C P}_{p^{m}}(n), f(\mathbf{x})$ задает n-квазигруппу на $\mathbb{Z}_{p^{m}}$, то любой ее парастроф ${ }^{\sigma} f \in \mathcal{Q C P}_{p^{m}}(n)$.

Ранее было сказано, что при $m=2$ имеет место равенство классов полиномиальных, ВКП и квази-ВКП-функций, т. е.

$$
\mathcal{P}_{p^{2}}(n)=\mathcal{C} \mathcal{P}_{p^{2}}(n)=\mathcal{Q} \mathcal{C} \mathcal{P}_{p^{2}}(n)
$$

В совокупности со следствием это приводит к следующему результату, который сформулируем в виде утверждения.

Утверждение 17. Если $\left(\mathbb{Z}_{p^{2}}, f(\mathbf{x})\right)$ - полиномиальная n-квазигруппа, то любой ее парастроф ${ }^{\sigma} f$ полиномиален. В частности, любая обратная операция полиномиальной п-квазигруппьл $\left(\mathbb{Z}_{p^{2}}, f(\mathbf{x})\right)$ полиномиальна.

В заключение параграфа докажем теорему о достаточном условии равновероятности ВКП-функции, обобщающую утверждение 5.

Теорема 18. Если $f(\mathbf{x}) \in \mathcal{C P}_{p^{m}}(n)$ имеет координатные многочлены $p_{0}(\mathbf{x}), \ldots, p_{m-1}(\mathbf{x})$ и для них выполнены следующие условия:

1) $p_{0}(\mathbf{x})(\bmod p)-$ равновероятная функичя над полем $\mathcal{B} ;$ 
2) $\operatorname{grad} p_{j}(\alpha) \not \boldsymbol{\theta}(\bmod p)$ при любых $\alpha \in \mathcal{B}^{n}, j \in \overline{1, m-1}$, где $\boldsymbol{\theta}$ - нулевой вектор,

то функиия $f(\mathbf{x})$ равновероятная.

Доказательство. Пусть $y \in \mathbb{Z}_{p^{m}} ;$ рассмотрим уравнение

$$
f(\mathbf{x})=y
$$

и докажем, что оно имеет в точности $p^{m(n-1)}$ решений. Решим его методом покоординатной линеаризации.

Приводя уравнение по модулю $p$, получим сравнение

$$
p_{0}\left(\mathbf{x}^{(0)}\right) \equiv y^{(0)}(\bmod p) .
$$

Поскольку $p_{0}(\mathbf{x})(\bmod p)-$ равновероятная функция от $n$ переменных над полем $\mathcal{B}$, постольку оно имеет $p^{n-1}$ решений.

Предположим, что при $j \in \overline{1, m-1}$ найдены значения координат неизвестных $\mathbf{x}^{(0)}=\mathbf{c}^{(0)}, \ldots, \mathbf{x}^{(j-1)}=\mathbf{c}^{(j-1)}$. Приведем уравнение по модулю $p^{j+1}$ и согласно алгоритму из доказательства теоремы 10 придем к сравнению:

$$
\operatorname{grad} p_{j}\left(\mathbf{c}^{(0)}\right) \cdot \mathbf{x}^{(j) \downarrow} \equiv y^{(j)} \ominus \gamma_{j} f_{i}\left(\mathbf{c}^{(0)}, \ldots, \mathbf{c}^{(j-1)}\right)(\bmod p) .
$$

Так как по условию $\operatorname{grad} p_{j}\left(\mathbf{c}^{(0)}\right) \not \boldsymbol{\theta}(\bmod p)$, то данное линейное сравнение от $n$ переменных над полем $\mathcal{B}$ имеет ровно $p^{n-1}$ решений.

Таким образом, при любом $j \in \overline{0, m-1}$ число $j$-х координат неизвестных равно $p^{n-1}$, а отсюда следует, что общее число решений такого уравнения равно $\left(p^{n-1}\right)^{m}=p^{m(n-1)}$ и, стало быть, функция $f(\mathbf{x})$ равновероятна.

Пример 6. Пусть ВКП-функция $f(\mathbf{x})$ имеет в качестве координатных многочленов аффинные функции над кольцом $\mathbb{Z}_{p^{m}}$ :

$$
p_{j}(\mathbf{x})=a_{j 1} x_{1}+\ldots+a_{j n} x_{n}+a_{j}, a_{j i}, a_{j} \in \mathbb{Z}_{p^{m}}, i=\overline{1, n}, j=\overline{0, m-1} .
$$

Аффинная функция $p_{0}(\mathbf{x})(\bmod p)$ равновероятна над полем $\mathcal{B}$ тогда и только тогда, когда среди коэффициентов $a_{01}, \ldots, a_{0 n} \in \mathbb{Z}_{p^{m}}$ есть хотя бы один обратимый. Это равносильно равновероятности функции $p_{0}(\mathbf{x})$. При 
$j \in \overline{1, m}$ градиент аффинной функции $p_{j}(\mathbf{x})$

$$
\operatorname{grad} p_{j}(\mathbf{x}) \equiv\left(a_{j 1}^{(0)}, \ldots, a_{j n}^{(0)}\right)(\bmod p)
$$

не сравним с $\boldsymbol{\theta}$ по модулю $p$ тогда и только тогда, когда среди коэффициентов $a_{j 1}, \ldots, a_{j n} \in \mathbb{Z}_{p^{m}}$ есть хотя бы один обратимый. Таким образом, получаем достаточное условие равновероятности ВКП-функции, имеющей аффинные координатные многочлены: если каждая из аффиных функций равновероятна, то равновероятна и ВКП-функция. Например, ВКП-функция над $\mathbb{Z}_{8}$ с координатными многочленами

$$
p_{0}(x, y)=x+y, p_{1}(x, y)=3 x+2 y, p_{2}(x, y)=4 x+5 y
$$

равновероятна и при этом не полиномиальна по теореме 7.

Даже в случае аффинных координатных функций полученное условие не является необходимым для равновероятности ВКП-функции, как показывает следующий пример.

Пример 7. ВКП-функция над $\mathbb{Z}_{8}$ с координатными многочленами

$$
p_{0}(x, y)=x+y, p_{1}(x, y)=x+2 y, p_{2}(x, y)=4 x
$$

равновероятна, как легко непосредственно проверить (см. ее значения в табл. 4). В то же время функция $p_{2}(x, y)=4 x$ не равновероятна.

Таблица 4

\begin{tabular}{|c|c|c|c|c|c|c|c|c|}
\hline $\mathbf{7}$ & 3 & 6 & 1 & 4 & 3 & 6 & 1 & 4 \\
\hline $\mathbf{6}$ & 0 & 5 & 2 & 7 & 0 & 5 & 2 & 7 \\
\hline $\mathbf{5}$ & 3 & 6 & 1 & 4 & 3 & 6 & 1 & 4 \\
\hline $\mathbf{4}$ & 0 & 5 & 2 & 7 & 0 & 5 & 2 & 7 \\
\hline $\mathbf{3}$ & 3 & 6 & 1 & 4 & 3 & 6 & 1 & 4 \\
\hline $\mathbf{2}$ & 0 & 5 & 2 & 7 & 0 & 5 & 2 & 7 \\
\hline $\mathbf{1}$ & 3 & 6 & 1 & 4 & 3 & 6 & 1 & 4 \\
\hline $\mathbf{0}$ & 0 & 5 & 2 & 7 & 0 & 5 & 2 & 7 \\
\hline$y / x$ & $\mathbf{0}$ & $\mathbf{1}$ & $\mathbf{2}$ & $\mathbf{3}$ & $\mathbf{4}$ & $\mathbf{5}$ & $\mathbf{6}$ & $\mathbf{7}$ \\
\hline
\end{tabular}

Данный пример также показывает, что условия теоремы 18 для равновероятности ВКП-функции не являются необходимыми.

\section{Список литературы}

1. Анашин B. С. Равномерно распределенные последовательности целых $p$ адических чисел // Дискретная математика. - 2002. - Т. 1. № 4. - С. 3-64. 
2. Anashin V.S. Uniformly distributed sequences in computer algebra or how to construct random number generators // J. Math. Sci. (Plenum Publishing Corp., New York). - 1998. - V. 89. № 4. - P. 1355-1390.

3. Lausch H., Nöbauer W. Algebra of Polynomials. - North-Holland Publ. Co, Amsterdam, 1973.

4. McDonald B. R. Finite Rings with Identity. - New York: Marcel Dekker, 1974.

5. Nöbauer $W$. Zur Theorie der Polynomtransformationen und Permutationspolynome // Math. Ann. - 1964. - V. 157. - P. 332-342.

6. Samardziska S., Markovski S. Polynomial $n$-ary quasigroups // Math. Maced. - 2007. - V. 5. - P. 77-81.

7. Zhang $Q$. Polynomial functions and permutation polynomials over some finite commutative rings // J. Number Theory. - 2004. - V. 105. - P. 192-202.

8. Белоусов В. Д. Основы теории квазигрупп. - М.: Наука, 1967.

9. Белоусов В.Д. n-арные квазигруппы. - Кишинев: Штиинца, 1972. $225 \mathrm{c}$.

10. Заеи М. В. Решение систем ВКП-уравнений методом покоординатной линеаризации над примарным кольцом вычетов. //Тезисы XLI Междунар. конф. и XI Междунар. конф. молодых ученых «Инф. технол. в науке, образов., телекоммун. и бизнесе IT $+\mathrm{SE} 13 » .-$ Вестник Московского ун-та им. С. Ю. Витте. - 2013. - Сер. 1 (приложение). - С. 155-157.

11. Заеи М. В., Никонов В. Г., Шишков А. Б. Класс функций с вариационнокоординатной полиномиальностью над кольцом $\mathbb{Z}_{2^{m}}$ и его обобщение. // Матем. вопросы криптографии. - 2013. - Т. 4. № 3. - С. 19-45.

12. Заеи М. В., Никонов В.Г., Шишков А. Б. Функции с вариационнокоординатной полиномиальностью и их свойства // Открытое образование. - 2012. - № 3. - С. 57-61.

13. Нечаев А. А. Полиномиальные преобразования конечных коммутативных локальных колец главных идеалов // Мат. заметки. - 1980. - Т. 27. Вып. 6. - С. 885-899.

14. Заеи М. В. О классе вариационно-координатно полиномиальных функций над примарным кольцом вычетов // ПДМ. - 2014. - № 3. - С. 12-28.

15. Заеи $M$. В. Координатно-линейно разрешимые функции над примарным кольцом вычетов и метод покоординатной линеаризации // Сетевой научный журнал «Образовательные ресурсы и технологии» - 2014. № 2(5). - C. 59-72. 